\title{
The Influence of Self-Threats on Fairness Judgments and Affective Measures
}

\author{
Joost Miedema, ${ }^{1,4}$ Kees van den Bos, ${ }^{2}$ and Riël Vermunt ${ }^{3}$
}

\begin{abstract}
This paper focuses on the question of why fairness matters to people. Extending on terror and uncertainty management theories and the literature on the self, it is proposed here that fairness can be a means of self-defense. Thinking of a situation that is threatening to the self therefore should make fairness a more important issue to people. The findings of two experiments support this line of reasoning: Asking participants to think about things that are threatening to themselves led to stronger reactions to manipulations of both procedural and distributive fairness. In the discussion it is argued that these findings suggest that fairness especially matters to people when they are trying to deal with threats to their selves.
\end{abstract}

KEY WORDS: self; fairness; fairness judgments; affective reactions.

\section{INTRODUCTION}

The norms and values of fairness and justice constitute a fundamental feature of human life. The issue of justice has received substantial attention from diverse scientific domains, such as philosophy, politics, sociology, law, economy, and psychology, among others. Social psychological research has shown convincingly that when people feel they have experienced a fair or

\footnotetext{
${ }^{1}$ Department of Social and Organizational Psychology, Leiden University, Leiden, The Netherlands

${ }^{2}$ Department of Social and Organizational Psychology, Utrecht University, Utrecht, The Netherlands

${ }^{3}$ Department of Psychology, Skövde University, Skövde, Sweden

${ }^{4}$ Address correspondence to: Joost Miedema, Faculty of Management and Organization, University of Groningen, P.O. Box 8009700 AV Groningen, The Netherlands, e-mail: j.l.miedema@rug.nl
} 
unfair event this may strongly influence their subsequent reactions (see, e.g., Brockner and Wiesenfeld, 1996; Folger and Cropanzano, 1998; Lind and Tyler, 1988; Tyler and Lind, 1992; Van den Bos and Lind, 2002). This indicates that perceived fairness is a crucial factor in social behavior. As Folger (1984) put it: "the importance of justice cannot be overstated" (p. ix). It is therefore imperative to study why and when fairness matters to people (Tyler, 1990, 1997).

In several reviews of the justice literature it has been suggested that in order to understand the frequently replicated effects of perceived fairness, such as the voice effect (see, e.g., Folger, 1977; Folger et al., 1979; Van den Bos et al., 1997), fair process effects (see, e.g., Folger and Cropanzano, 1998; Lind and Tyler, 1988; Van den Bos and Lind, 2002), or fair outcome effects (see, e.g., Adams, 1965; Van den Bos 1999) on people's reactions we have to thoroughly study the psychological processes underlying these effects (Folger and Cropanzano, 1998; Lind and Tyler, 1988; Tyler and Lind, 1992; Van den Bos and Lind, 2002). In this paper, we try to do this by focusing on the question of why fairness matters to people (cf. Tyler, 1990, 1997). More specifically, earlier research has shown that self-related threats, such as salience of personal uncertainties (see, e.g., Van den Bos, 2001; Van den Bos et al., 2005a) and reminders of one's own mortality (see, e.g., Van den Bos and Miedema, 2000), can have a profound influence on people's perceptions of, and reactions toward fairness. We argue here that these results are indicative of why and when fairness matters to people, because fairness may provide people with a means to re-establish a positive self-evaluation. After experiencing a self-threatening event, people may try to reinforce their selves by falling back on something else (see also Tesser, 2000): fairness is a universally accepted social rule, and may therefore be used as a tool to reestablish their self (for a more elaborate explanation, see below).

Fairness judgments are subjective judgments (cf. Mikula and Wenzel, 2000; Van den Bos and Lind, 2002). This subjective idea-sometimes feeling - of what is and is not fair, is not the same as objective, normative descriptions of fairness and justice as put forward by, for instance, philosophers or economists (see, e.g., Cohen, 1986; see also Van den Bos and Lind, 2002). The subjectivity of fairness could well lead to changes in what is considered fair: Not only could fairness judgments vary from person to person, but a person may well evaluate the fairness of one allocation event differently from the fairness of another event depending on the specific characteristics of the allocation event. In the current paper, we aim to further our insights into the issue of why fairness matters to people (cf. Tyler, 1990, 1997) by directly examining the effects of threats that are directly related to people's selves. To investigate this proposed strong relationship between the concepts of self and fairness, the current studies were designed to elicit responses that are focused on the participants' own outcome or procedure. 
To this end, we will explore whether different threats to the self influence reactions toward both voice and no-voice procedures (Experiment 1) and equal and unequal outcomes (Experiment 2). We will now briefly outline why we think this research is needed and why previous work on the self and self-enhancement is important in the empirical study of why and when fairness matters to people.

\section{HOW FAIRNESS JUDGMENTS ARE FORMED: A CLOSER LOOK AT THE SELF}

Achieving a positive self-evaluation is one of the most important motivations in social psychology (Baumeister, 1998; Koole, 2000; Tesser and Martin, 1996). Previous research on justice and fairness has shown that the social psychology of fairness judgments is a self-related process. Fairness, assessed with an interactional justice scale, has a positive influence on people's affective commitment to their organization - but this is only the case when employees have low self-esteem (De Cremer et al., 2004). Smith et al. (1998) showed that when people are treated fairly by an authority, they show higher levels of state self-esteem. These authors further found that this effect is enhanced when the authority is important to them (e.g., belongs to their ingroup). Koper et al. (1993) also studied the effects of fair and unfair procedures on self-esteem. Their experiments showed that people reported having lower levels of state self-esteem after an unfair procedure than after a fair procedure. Koper et al. (1993) explained that evaluations of fairness are influenced by self-reflective evaluations: People see or evaluate themselves in accordance with how they think others see or evaluate them ("they think I'm great, so I must be great"). Vermunt et al. (2001) found that people with low self-esteem are more influenced by variations in procedural justice than people with high self-esteem. Brockner et al. (1998) found that high selfesteem persons are more affected by their perceived level of voice than low self-esteem persons (we will return to these seemingly conflicting results in the General Discussion; see also Van den Bos et al., 2005b). Thus, fairness can have a profound influence on how people think and feel about themselves (see, e.g., Koper et al., 1993; Smith et al., 1998), and self-esteem can have considerable effects on how fairness judgments are formed. We argue here that these results can be interpreted as providing tentative support for what we currently propose as the underlying psychological process: fairness can be conceived of as a means of self-maintenance (cf. Tesser, 2000).

There are at least two important lines of research that also have addressed the importance of fairness and the function it provides for people. The research on terror management theory (e.g., Van den Bos and Miedema, 2000) and the uncertainty management model (Van den Bos and Lind, 2002; 
Van den Bos et al., 2005a) clearly shows that fairness becomes more important to people when they feel threatened by, for instance, thoughts of their own mortality (Van den Bos and Miedema, 2000) or a lack of control over a situation (Van den Bos, 2001).

Terror management theory (for overviews, see, Greenberg et al., 1997; Pyszczynski et al., 1999; Solomon et al., 1991) proposes that people are aware of the inevitability of death, and that this creates the potential for terror. This terror, in turn, is managed by a cultural anxiety buffer, a social psychological construct that consists of things like one's worldview and selfesteem. As the cultural anxiety buffer protects people against death concerns, a reminder of their mortality increases people's need for that buffer. That is, reminders of death should lead to strong negative evaluations of persons whose behaviors or beliefs impinge on the cultural worldview, and lead to strong positive evaluations of persons who uphold or bolster the worldview. A more elaborate introduction to terror management theory and the supporting empirical findings is beyond the scope of the current paper, (for more complete descriptions, see e.g., Arndt et al., 1999; Greenberg et al., 1990; Harmon-Jones et al., 1997; Pyszczynski et al., 1999; Rosenblatt et al. 1989; Solomon et al., 1991), but it should be noted here that results are in agreement with the theory's line of reasoning.

The uncertainty management model (Van den Bos, 2001; Van den Bos and Lind, 2002) states that because the world is an uncertain place and other people are unpredictable, people use fairness as a means to cope with uncertainties in their daily lives. The model argues that people have a fundamental need to feel certain about the world and their place in it, that uncertainty can be threatening, and that people feel a need to reduce, eliminate, or somehow manage this uncertainty (Van den Bos et al., 2005b). This model further proposes that the experience of fairness can reduce uncertainty by making the uncertainty more tolerable, or by making things seem more certain. Experimental results (Van den Bos, 2001; Van den Bos, et al., 2005a) indicate that when people feel uncertain, or when they think of uncertain aspects of their world, they indeed react quite strongly to fair and unfair events.

We note here that the above-mentioned studies on fairness, mortality salience and personal uncertainty refer to self-related processes. The will to think positively of oneself is one of the most fundamental and widespread motivations in human psychological functioning (Leary and Baumeister, 2000) and negative information therefore poses a threat to the self. Personal uncertainty (see, e.g., Van den Bos, 2001; Van den Bos et al., 2005a) and own mortality concerns (Van den Bos and Miedema, 2000) can easily be experienced as threats to people's self-concepts and people want to protect themselves against these threats. What we propose here is that mortality and uncertainty have those profound effects on perceived fairness, because they 
are threatening to the self and thus motivate people to make use of selfesteem maintenance mechanisms (Tesser, 2000). Fairness may in this light be seen as a means of self-esteem maintenance, as it provides people with a strong and often effective argument to manage self-threatening situations.

\section{THE CURRENT RESEARCH}

In the current paper, two experiments will be presented. In the first experiment, we will explore whether a self-threat other than mortality or uncertainty can yield comparable effects as those reported in earlier research (e.g., Van den Bos, 2001; Van den Bos and Miedema, 2000). If self-threats are indeed important in understanding why fairness matters, directly inducing a self-threat should lead to stronger reactions to perceived fairness. The idea that fairness can be seen as a means by which people can manage or handle self-threats needs to be tested against (and compared with) other good explanations for why people are interested in fairness. There are indications (see, e.g., McGregor et al., 2001; Mikulincer et al., 2002) that mortality salience manipulations are rather broad, and can evoke not only thoughts of someone's own mortality, but of other topics as well, such as uncertainty, or concerns about how others will cope with the loss. Therefore, in the second experiment to be presented here, we will compare one threat to the self to another type of self-threat (mortality salience) to see whether these two concepts result in effects of comparable or different magnitude.

\section{EXPERIMENT 1}

On the basis of the line of reasoning outlined above, it was argued in Experiment 1 that a threat to the self would lead participants to show variations in their reactions toward perceived fairness. Thus, it was predicted that a self-threat would evoke stronger fair process effects than no selfthreat: We expected that, compared to the control condition, after a selfthreat a voice procedure would be judged to be more fair, and a no-voice procedure would be judged as less fair.

In Experiment 1, following and expanding on previous terror management and uncertainty management studies (for overviews, see Greenberg et al., 1997; Pyszczynski et al., 1999; Solomon et al., 1991; Van den Bos, 2001, respectively), the self-threat condition was induced by having participants write down their responses to two questions about their being perceived as being not intelligent. The reason behind this was that, because all our participants were university students, intelligence is a trait that can be assumed to be very central to the participants' selves. The no-threat 
condition was induced by having participants write down their responses to two questions about being perceived as intelligent. The fairness manipulation of Experiment 1 consisted of the best-documented and most generally accepted manipulation in procedural justice experiments: Participants received or did not receive an opportunity to voice their opinion about a decision (cf. Van den Bos et al., 1997; Van den Bos and Miedema, 2000). Following previous studies (e.g., Folger, 1977; Stroessner and Heuer, 1996; Van den Bos et al., 1996), the dependent variables of this experiment were ratings of perceived procedural fairness.

\section{Method}

\section{Participants and Design}

Sixty-two students (eight men and 54 women) at Leiden University participated in the experiment and were paid for their participation. Participants were randomly assigned to one of the conditions of the 2 (salience: self-threat vs. no-threat) $\times 2$ (procedural fairness: voice vs. no voice) factorial design. Fifteen to sixteen participants took part in each of the conditions.

\section{Experimental Procedure}

Students at Leiden University were invited to the laboratory to participate in a study on how people perform tasks. On arrival at the laboratory, participants were asked to take a seat in separate cubicles, each of which contained a computer with a keyboard. Participants were told that all instructions would be given by means of the computer. Next to the monitor, participants found a piece of paper and a pencil. Participants were told that the computers were connected to a computer network and that the experimenter could communicate with them by means of this network. The computers were used to present the stimulus information and to collect data on the dependent variables and the manipulation checks. Participants participated in the experiment after participating in another, unrelated experiment. The experiments lasted a total of $100 \mathrm{~min}$ and participants were paid 17.50 Dutch guilders for their participation (one Dutch guilder equals approximately $€ 0.45$, and equaled approximately $\$ 0.45$ U.S. at the time the experiments presented here were conducted).

In the first part of the instructions participants were informed that they participated in the experiment with another person, referred to as Other. The experimental procedure was then explained to the participants. After an explanation of the experimental tasks, participants were to practice the tasks 
for two minutes, after which they would work on the tasks for $10 \mathrm{~min}$. Participants were also informed that after all participants were run, a lottery would be held among all participants. The winner of this lottery would receive 100 Dutch guilders (actually, after all participants had completed the experiment, the 100 Dutch guilders were randomly given to one participant; none of the participants objected to this experimental procedure upon debriefing). Participants were told that a total of 200 lottery tickets would be divided among all participants. Furthermore, participants were told that after the work round the experimenter would divide some lottery tickets between them and Other. Seven practice questions were asked to ensure comprehension of the lottery. If a participant gave a wrong answer to a question, the right answer was shown and the main characteristics of the lottery were repeated.

The task was then explained to the participants. The task was the same as in the studies conducted by Van den Bos and his colleagues (e.g., Van den Bos et al., 1997): Figures would be presented on the upper right part of the computer screen. Each figure consisted of 36 squares and each square showed one of eight distinct patterns. On the upper left side of the screen one of the eight patterns would be presented. Participants had to count the number of squares with this pattern in the figure on the right side of the screen. After indicating the correct number of patterns in the figure, another figure and another pattern were presented to the participants. In both the practice round and the work round, the number of tasks a participant had completed (the number of figures that the participant had counted) in the present round was presented on the lower right side of the screen. The time remaining in the present round was presented on the lower left side of the screen.

The practice round and the following work round then took place. After the work round had ended, the participants were told the number of tasks they had completed in the work round (cf. Van den Bos et al., 1997). In order to ensure that participants compared themselves to Other, the participants were informed that Other had completed an equivalent number of tasks. To assess whether participants thought of Other as a person who was comparable in the amounts of inputs he or she provided, they were asked to what extent Other had performed well in the work round relative to the performance of the participants themselves $(1=$ much worse, $4=$ equally, $7=$ much better), to what extent Other did his or her best in the work round relative to the participants themselves $(1=$ much worse, $4=$ equally, $7=$ much better $)$, and to what extent Other was good in performing the tasks in the work round relative to the participants themselves $(1=$ much worse, $4=$ equally, $7=$ much better $)$. After this, participants were asked to think for one minute about the percentage of lottery tickets that they should receive relative to Other. 
Participants were then informed that before the experimenter would divide the lottery tickets between them and Other, they would be asked to complete two questionnaires and that after the questionnaires were completed, the study would continue. Self-threat salience was manipulated by means of the first questionnaire. Following previous studies on terror management theory (e.g., Van den Bos and Miedema, 2000) and the uncertainty management model (e.g., Van den Bos, 2001) self-threat salience was manipulated by having participants respond to two questions and a scale measuring how participants felt at the moment. In the selfthreat salient condition, participants were asked to write down their answers to the questions (1) "Please briefly describe the emotions that arise in you when you think of other people believing you are not at all intelligent" and (2) "Please briefly describe the emotions that arise in you when you think of an actual situation in which you did not act intelligently at all." Participants in the no-threat salient condition were asked to write down their answers to the questions (1) "Please briefly describe the emotions that arise in you when you think of other people believing you are intelligent" and (2) "Please briefly describe the emotions that arise in you when you think of an actual situation in which you acted intelligently."

After this, all participants completed the Positive and Negative Affect Schedule (PANAS; Watson et al., 1988), on which they reported on 20 items how they felt at the moment. Following previous terror and uncertainty management studies (see Greenberg et al., 1997; Solomon et al., 1991; Van den Bos, 2001; Van den Bos et al., 2005a; Van den Bos and Miedema, 2000), we included the PANAS as a filler task and to determine whether the manipulation of self-threat engendered positive and negative affect. The PANAS consists of two ten-item subsets (Watson et al., 1988), one measuring positive affect (PA) and one measuring negative affect (NA), and both subsets were averaged to form reliable scales $(\alpha \mathrm{s}=0.86$ and 0.70 , respectively). After completion of the PANAS, participants were told that by pushing the return button on the keyboard the study would continue.

Then, procedural fairness was manipulated. In the voice condition, the experimenter allegedly asked participants, by means of the computer network, to type in their opinion about the percentage of tickets that they should receive relative to Other. (In reality, however, all stimulus information was preprogrammed). Participants in the no-voice condition were informed that they would not be asked to type their opinion about the percentage of tickets that they should receive relative to Other.

After this, participants were asked questions pertaining to the dependent variables and manipulation checks. All ratings were made on 7-point scales. Participants' ratings of procedural fairness were assessed by asking participants how fair $(1=$ very unfair, $7=$ very fair $)$ and 
justified $(1=$ not at all justified, $7=$ very justified $)$ they thought the procedure they received was and how biased they thought the experimenter was $(1=$ not at all biased, $7=$ very biased $)$. After recoding the last item, these three dependent variables were averaged to form a reliable scale of perceived fairness $(\alpha=0.92)$. To check whether the voice procedure manipulation had been induced as intended, participants were asked to what extent they agreed with the statement that they had been given an opportunity to voice their opinion about the percentage of tickets that they should receive relative to Other $(1=$ strongly disagree, $7=$ strongly agree) and to what extent they agreed with the statement that they had not been given an opportunity to voice their opinion about the percentage of tickets that they should receive relative to Other $(1=$ strongly disagree, $7=$ strongly agree). (Following previous work on terror management (e.g. Arndt et al., 1999; Greenberg et al., 1990) and uncertainty management (e.g., Van den Bos, 2001) the manipulations of self-threat were not explicitly checked, but debriefing interviews and checking what participants wrote down during the self-threat salience manipulation indicated that this manipulation was induced as intended. Furthermore, results to be presented below show that this manipulation had the intended effects on the dependent variables.) When the participants had answered these questions, they were thoroughly debriefed, paid and thanked for their participation.

\section{Results}

\section{Manipulation Checks}

A two-way multivariate analysis of variance (MANOVA) on the two manipulation checks of the procedure manipulation yielded only a main effect of procedure at both the multivariate level and the univariate levels, multivariate $F(2,54)=79.81, p<0.001$; for the voice check, $\quad F(1,58)=77.24, \quad p<0.001$; for the no-voice check, $F(1$, $58)=160.15, p<0.001$. Inspection of the means indicated that participants in the voice condition agreed more with the statement that they received an opportunity to voice their opinion $(M=5.8, \quad \mathrm{SD}=1.5)$ than participants in the no-voice condition $(M=2.0, \mathrm{SD}=1.8)$. Participants in the no-voice condition agreed more with the statement that they did not receive an opportunity to voice their opinion $(M=6.1$, $\mathrm{SD}=1.5)$ than did the participants in the voice condition $(M=1.8$, $\mathrm{SD}=0.9$ ). This indicates that the procedure manipulation was successfully operationalized. 


\section{PANAS Findings}

The PANAS questionnaire was included as a filler task, and to find out whether self-threat had an effect on participants' affective reactions. In line with most terror- and uncertainty management studies, such as Van den Bos and Miedema (2000), or Van den Bos et al. (2005a), the PANAS questionnaire was administered immediately after the self-threat manipulation and before the manipulation of procedure. As expected, a two-way multivariate analysis of variance (MANOVA) yielded no significant effects on participants' scores on the PANAS scales (PA scale $M=3.1, \mathrm{SD}=0.7$; NA scale $M=1.4, \mathrm{SD}=0.4)$. This suggests that the self-threat manipulation alone had no effects on participants' affective states.

\section{Salience Manipulation Responses}

In the threat conditions, participants were asked to write down their responses when they thought of their being perceived as not at all intelligent. To assess whether these questions did not lead participants to think about death-related issues or general uncertainty, two judges coded whether the answers that participants wrote down had anything to do with mortality or uncertainty. Independently of each other the judges agreed that none of the answers were related to mortality. Furthermore, the judges agreed that in both salience conditions some participants wrote down answers about self-related uncertainty: In the self-threat conditions four participants did so, and in the no-threat conditions three participants. Removing the data of these participants did not change the conclusions drawn from the dataset, and therefore data from these participants were not removed. These findings indicate that, as expected, general uncertainty or death-related thoughts cannot explain the findings reported here.

\section{Comparability Measures}

Participants' comparability judgments yielded no significant effects at either the multivariate level or the univariate level. Participants indicated that the other participant had performed equally well in the work round $(M=3.9, \mathrm{SD}=0.5)$, that the other participant equally did his or her best in the work round $(M=3.9, \mathrm{SD}=0.5)$ and that the other participant was equally good in performing the tasks in the work round $(M=4.0$, $\mathrm{SD}=0.4$ ). These means did not differ significantly from " 4 ", the midpoint of the scale. 


\section{Percentage Findings}

Participants who were allowed voice $(n=32)$ typed in their opinion about the percentage of lottery tickets that they should receive relative to the other participant. An analysis of variance (ANOVA) yielded no significant effect of the self-threat manipulation. Inspection of the means indicated that participants typed in that the lottery tickets should be equally divided between themselves and the other participant: the mean percentage $(M=52.7$, $\mathrm{SD}=18.3$ ) did not differ significantly from $50 \%$. These findings corroborate equity theory (cf. Adams, 1965): Participants preferred to divide outcomes equally between themselves and the other participant (who contributed an equal amount of inputs and hence deserved - according to equity theory - to receive the same amount of outcomes as the participants themselves).

\section{Dependent Variables}

Main dependent variables in this experiment were participants' ratings of fairness. The means and standard deviations of the procedural fairness scale are displayed in Table I. A two-way MANOVA showed a main effect of the procedure manipulation, $F(1,58)=47.47, p<0.001$, indicating that participants' ratings of fairness were higher in the voice conditions than in the no-voice conditions. This main effect was qualified by the predicted significant interaction between procedural fairness and self-threat salience, $F(1,58)=11.09, p<0.005$. In accordance with our predictions, the effect of procedure was stronger in the self-threat salient condition, $F(1,58)=52.32$, $p<0.001, \eta^{2}=0.47$, than in the no-threat salient (control) condition, $F(1,58)=6.29, p<0.02, \eta^{2}=0.10$.

It can also be noted that significant effects of salience were found within the voice as well as the no-voice conditions. The effect of self-threat within

Table I Means and Standard Deviations of Perceived Fairness as a Function of Salience and Procedure Manipulations

\begin{tabular}{llllll}
\hline & \multicolumn{3}{c}{ Salience } \\
\cline { 2 - 6 } & \multicolumn{2}{c}{ Self-threat } & & \multicolumn{2}{c}{ No self-threat } \\
\cline { 2 - 6 } Procedure & $M$ & SD & & $M$ & SD \\
\hline Voice & 4.9 & 0.8 & 4.2 & 1.1 \\
No voice & 2.7 & 0.7 & 3.4 & 0.8 \\
\hline
\end{tabular}

Note. Entries are means on 7-point scales, with higher values indicating higher ratings of perceived procedural fairness. 
the voice condition, $F(1,58)=6.59, p<0.02, \eta^{2}=0.10$, was slightly stronger than the effect within the no-voice condition, $F(1,58)=4.62$, $p<0.04, \eta^{2}=0.07$. We will further discuss these findings in the General Discussion.

\section{Discussion}

These findings support our line of reasoning: As expected, the effect of procedure was stronger in the self-threat salient condition than in the nothreat salient condition. The interaction effect we intended to find is driven by both a more positive reaction to voice procedures and a more negative reaction to no-voice procedures: In the self-threat conditions, people who received the opportunity to voice their opinion were more positive, and in the no-voice condition more negative in their fairness judgments, as compared to participants in the no-threat conditions. This suggests that under conditions of self-threat, people react stronger toward fair and unfair treatment. To explore whether different types of self-threats evoke different reactions, a second experiment was necessary.

\section{EXPERIMENT 2}

In Experiment 1 we used a manipulation of procedure as our fairness manipulation. Because fairness constitutes not only the process leading to outcome distributions, but the actual outcome distribution as well, it was important to show that similar results could be found using a manipulation of distributive justice. Participants in Experiment 2 were therefore informed that they received the same outcome as a comparable other or a worse outcome than a comparable other. The same argument goes for the manipulation of self-threat. In order to see whether this manipulation has robust effects, we used a different manipulation of self-threat: Participants in the threat conditions were asked to write down their responses to two questions about being judged by others in a negative way.

Furthermore, we will investigate whether different self-threats influence reactions to fairness to the same extent. Previous research suggests that uncertainty salience (Van den Bos, 2001; Van den Bos et al., 2005a) has a greater impact on people's reactions to variations in fairness manipulations than did mortality salience. For example, the five experiments reported by Van den Bos et al. (2005a) consistently showed that uncertainty salience had a bigger impact on people's fairness-related reactions than mortality salience. This would suggest that uncertainty could be a more important factor in predicting behavior, related to cultural norms and values such as fairness, 
than mortality salience. These studies further indicated that reactions to norms and values were stronger among mortality salient participants who, as a result of the mortality salience manipulation, thought of uncertainty than mortality salient participants who did not think of uncertainty following this manipulation. Uncertainty salience did not instigate death-thoughts in these studies.

However, as Van den Bos and Lind (2002) pointed out in their discussion of the uncertainty management model, the conceptualization of uncertainty, as it was used in previous theorizing and empirical studies, was perhaps too vague. As these authors point out, there may be a wide range of uncertainties, which could vary greatly in importance and in level of uncertainty. We agree with Van den Bos and Lind (2002) that this concept needs to be specified. More specifically, in the current paper, we attempted to further specify the broad concept of uncertainty to information that is threatening to the self. Experiment 2 was intended to see whether a direct threat to the self could have a bigger impact on people's reactions toward a fair or unfair outcome than an important alternative concept (i.e., mortality salience).

To make sure the salience manipulations were induced as intended, we added extra manipulation checks. Not only were the written answers inspected by two independent judges to assess whether there were self-threat and mortality-related thoughts in the two salience conditions, we also further checked the salience manipulation by asking all participants to indicate on a 7-point scale whether they had been thinking of mortality or being judged in a negative way during the experiment.

As in Experiment 1, we constructed a threat salience manipulation that closely resembled the mortality salience manipulations most often used in terror management studies (for overviews, see, e.g., Greenberg et al., 1997; Pyszczynski et al., 1999; Solomon et al., 1991). Following previous terror management studies (cf. Arndt et al., 1999; Harmon-Jones et al., 1997; Van den Bos and Miedema, 2000) the mortality salient condition was induced by asking participants two open-ended questions concerning their thoughts and feelings about their death. Participants in the self-threat salient conditions were asked two questions that were similar in format but that asked participants about their thoughts and feelings of their being judged in a negative way. The self-threat salience manipulation was constructed such that it closely resembled the mortality salience manipulation and that, as a result, the effects of these manipulations on people's responses toward fair and unfair outcomes could be investigated in a way that scientifically made sense.

In Experiment 2 we used two dependent variables: the scale of perceived fairness, which was similar to Experiment 1, and a scale of negative affect. Because it is important to measure affective reactions to perceived justice (Tyler and Smith, 1998), and following previous justice research (e.g., Folger 
et al., 1979; Van den Bos and Miedema, 2000) we added ratings of affect to our dependent variables. More specifically, because it is important to assess negative affect following perceived fairness (Folger and Cropanzano, 1998; Folger et al., 1979), and because findings of Van den Bos and colleagues (Van den Bos and Miedema, 2000; Van den Bos et al., 2005a) showed strong effects of mortality salience on participants' ratings of negative affect, we asked participants to what extent they were in a negative mood and to what extent they were in a hostile mood (cf. Van den Bos, 2001).

An additional aim of this second experiment was to use a less disruptive experimental procedure than in Experiment 1. In Experiment 1, participants read instructions, completed a series of tasks, and answered questions about another participant. Then the experiment was stopped, self-threat was manipulated, and the PANAS was administered. After completion of the PANAS, the experiment continued. To be able to control for possible effects of this procedure, in Experiment 2 we decided to use another experimental procedure. To achieve this aim, we presented the experiment as two unrelated studies. In the first study, we manipulated self-threat or mortality salience, after which participants filled out the PANAS. The second study was ostensibly unrelated to the first and in it participants received stimulus materials that included the manipulation of outcome and questions pertaining to the dependent variables and manipulation checks.

\section{Method}

\section{Participants and Design}

Eighty-three students (22 men and 61 women) at Leiden University participated in the experiment and were paid for their participation. Participants were randomly assigned to one of the conditions of the 2 (salience: self-threat vs. mortality) $\times 2$ (outcome: equal vs. unequal) factorial design. A minimum of nineteen and a maximum of 22 participants took part in each of the conditions.

\section{Experimental Procedure}

Students at Leiden University were invited to the laboratory. On arrival at the laboratory, participants were asked to take a seat in one of the separate cubicles, each of which contained a computer with a keyboard. Next to the monitor participants found a piece of paper and a pencil. The experimenter informed the participants that all instructions would be given by means of the computer and left the cubicle. 
In the first part of the experiment, salience was manipulated. This manipulation was again induced by asking the participants two questions. Participants in the self-threat salient conditions were asked to write down on a piece of paper their answer to the questions (1) "Please briefly describe the emotions that arise in you when you think of other people judging you in a very negative way" and (2) "Please briefly describe the emotions that arise in you when you think of an actual situation in which you were judged very negatively." Following previous terror management studies (e.g. Arndt et al., 1999; Greenberg et al., 1990; Van den Bos and Miedema, 2000), participants in the mortality salient conditions were asked to write down on their piece of paper their answers to the questions (1) "Please briefly describe the emotions that the thought of your death arouses in you" and (2) "Please write down, as specifically as you can, what you think will happen to you as you physically die."

After this, all participants completed the PANAS (Watson et al., 1988). Both the ten-item positive affect subset (PA) and the ten-item subset of negative affect (NA) were averaged to form reliable scales $(\alpha=0.82$ and 0.89 , respectively).

After filling out the PANAS, participants read the scenario. They were asked to imagine the following situation:

\begin{abstract}
Together with a fellow student, you have had a summer job. The two of you were working as a pair. The organization you worked for has a lot of employees working in pairs. You and your fellow student have worked equally hard and have performed equally well. Because the organization has done very well that summer, it is announced at the last workday of the summer that a bonus of 10,000 Dutch guilders will be divided amongst all employees. A certain amount of bonus money has been set aside for your pair (you and your fellow student). Your supervisor still has to decide how the money, reserved for you and your fellow student, will be divided. A week later, payment of the bonus money takes place.
\end{abstract}

This was followed by the manipulation of outcome. Participants read the sentence (manipulated information is in italics):

You receive 450 Dutch guilders; Your fellow student receives $450 / 900$ Dutch guilders.

After this we solicited the dependent variables. We assessed the same scale of perceived fairness as in Experiment 1, adapted to fit distributive justice: Participants were asked how just $(1=$ very unjust, $7=$ very just $)$ and how justified $(1=$ not at all justified, $7=$ very justified $)$ they thought their outcome was, and how biased they thought the supervisor was $(1=$ not at all biased, $7=$ very biased $)$. After recoding the last item, these three questions were averaged to form a reliable scale of perceived fairness $(\alpha=0.93)$.

Furthermore, we asked participants questions pertaining to their affective state: Participants were asked to what extent they were in a negative $\operatorname{mood}(1=$ not at all, $7=$ very much so $)$ and to what extent they felt hostile $(1=$ not at all, $7=$ very much so $)$. These two questions were averaged to form a reliable scale of negative affect $(\alpha=0.85)$. To check whether outcome 
was manipulated as intended, participants were asked the amount of money they received and the amount of money the fellow student received. Finally, to check whether the salience manipulations were induced as intended, participants were asked whether they had been thinking about being judged negatively during the experiment, and whether they had been thinking about their mortality during the experiment. These two questions were both answered on 7-point scales, ranging from 1 (not at all) to 7 (very much so). A further check consisted of analyzing what participants wrote down during the salience manipulation. After answering all questions, participants were thoroughly debriefed, paid, and thanked for their participation.

\section{Results}

\section{Manipulation Checks}

Almost all participants answered the question about the amount of money they received $(450)$ correctly $(M=448.2, \mathrm{SD}=9.4)$; this mean did not differ significantly from 450. All participants answered the other question, pertaining to the amount of money the fellow student received, correctly (for the unequal outcome conditions $M=900, \mathrm{SD}=0.0$; for the equal outcome conditions $M=450, \mathrm{SD}=0.0$ ). This indicates that the manipulation of outcome was induced as intended.

A two-way MANOVA on the two manipulation checks of self-threat and mortality salience yielded only a main effect of salience at both the multivariate level and the univariate levels, multivariate $F(2,76)=78.36$, $p<0.001$; for the check of whether participants had thought about being judged negatively, $F(1,79)=12.96, p<0.001$; for the check of how much participants had thought about their mortality, $F(1,79)=140.74, p<0.001$. As expected, participants in the self-threat conditions thought more about being judged negatively $(M=3.8, \mathrm{SD}=2.0)$ and less about their mortality $(M=1.4, \mathrm{SD}=1.1)$ than participants in the mortality salience conditions $(M \mathrm{~s}=2.4$ and 5.4, SDs $=1.5$ and 1.8 , respectively $)$.

In the self-threat salient conditions, participants were asked to write down their responses when thinking of being judged very negatively. To assess whether this did not lead participants to think about death-related issues, two judges coded whether the answers, which had been written down by the participants, had anything to do with death. The two judges agreed, independently of each other, that all answers had nothing to do with death but with emotional responses to being judged negatively. Thus, death-related thoughts did not occur in the self-threat conditions. These findings are in line with Experiment 1 and those of Van den Bos et al. (2005a) and suggest that death-related thoughts cannot explain the effects within the self-threat 
salient conditions. The judges also checked whether the participants in the mortality salient conditions had written down any answers related to being judged in a negative way. The judges agreed that this was not the case: The answers described thoughts and feelings about mortality. This, too, suggests that the salience manipulation was induced as intended.

Furthermore, some uncertainty-related answers were given in both the self-threat and the mortality salient conditions. In the self-threat conditions, the answers were on thoughts and feelings about self-threats, but because self-threats evoke self-related uncertainty, 15 participants in the self-threat salient conditions reported not only threat-related feelings and thoughts, but also some feelings of uncertainty. Interestingly, in the mortality salient conditions 12 participants also wrote down uncertainty-related answers. Removing the data of participants who had written down uncertainty-related answers did not lead to different conclusions, and therefore data of these participants were included in the analyses. Moreover, the manipulation checks of salience and the checks of the written answers indicate that salience was induced as intended.

\section{PANAS Findings}

As expected, participants' scores showed no significant effects at both the multivariate and the univariate levels. This shows that, in correspondence with earlier studies (e.g., Van den Bos and Miedema, 2000) and Experiment 1, salience had no effect on the PA scale $(M=2.8, \mathrm{SD}=0.7)$ or the NA scale $(M=1.3, \mathrm{SD}=0.5)$, and that, as expected, outcome had no effect on the PANAS scales (which were assessed before outcome was manipulated).

\section{Dependent Variables}

The main dependent variables in this experiment were participants' ratings of fairness and negative affect. The means of the two scales are displayed in Table II. Both scales were measured after salience and outcome had been induced. We first inspected the multivariate effects on these two scales. This yielded only a multivariate main effect of outcome, $F(2$, $76)=474.07, \quad p<0.001$, and a multivariate interaction effect, $F(2$, 76) $=4.55, p<0.02$, showing that the multivariate effect of outcome was stronger in the self-threat salient condition, $F(2,78)=50.65, p<0.001$, $\eta^{2}=0.56$, than in the mortality salient condition, $F(2,78)=25.03$, $p<0.001, \eta^{2}=0.39$. After this, we inspected the univariate effects on the two scales. 
Table II. Means and Standard Deviations of Perceived Fairness and Negative Affect as a Function of Salience and Outcome Manipulations

\begin{tabular}{|c|c|c|c|c|c|}
\hline & \multirow[b]{3}{*}{ Outcome } & \multicolumn{4}{|c|}{ Salience } \\
\hline & & \multicolumn{2}{|c|}{ Self-threat } & \multicolumn{2}{|c|}{ Mortality } \\
\hline & & $M$ & SD & $M$ & $\mathrm{SD}$ \\
\hline \multirow[t]{2}{*}{ Perceived fairness } & Equal & 6.4 & 0.6 & 6.0 & 0.8 \\
\hline & Unequal & 1.7 & 0.5 & 3.4 & 0.6 \\
\hline \multirow[t]{2}{*}{ Negative affect } & Equal & 1.3 & 0.5 & 1.7 & 1.5 \\
\hline & Unequal & 5.1 & 1.3 & 4.3 & 1.4 \\
\hline
\end{tabular}

Note. Entries are means on 7-point scales, with higher values indicating higher ratings of distributive justice or higher ratings of negative affect.

Participants' ratings of fairness showed only a main effect of outcome, $F(1,79)=960.28, p<0.001$, and an interaction effect, $F(1,79)=7.73$, $p<0.01$. The main effect of outcome indicated that participants rated receiving an equal outcome as more fair than receiving an unequal outcome. More interestingly, the interaction effect showed that the effect of outcome was stronger in the self-threat salient condition, $F(1,79)=99.51, p<0.001$, $\eta^{2}=0.55$, than in the mortality salient condition, $F(1,79)=48.60$, $p<0.001, \eta^{2}=0.38$. This is supportive of our line of reasoning.

Participants' ratings of negative affect yielded only a main effect of outcome, $F(1,79)=141.87, p<0.001$, and an interaction effect, $F(1,79)=4.78, p<0.04$. The main effect of outcome indicated that participants' ratings were less negative in the equal outcome condition than in the unequal outcome condition. The interaction effect showed that, as expected, the effect of outcome was stronger in the self-threat salient condition, $F(1,79)=64.96, p<0.001, \eta^{2}=0.45$, than in the mortality salient condition, $F(1,79)=21.48, p<0.001, \eta^{2}=0.21$. This, too, supports our expectations.

It can also be noted that for both dependent variables, the effects of the salience manipulation within both outcome conditions did not reach conventional levels of significance on either the multivariate or univariate levels.

\section{Discussion}

The results of Experiment 2 reveal that stronger effects of outcome were found after a self-threat than after mortality salience. These results thus support the current line of reasoning that threats to the self may be a major cause of people's reactions toward fairness manipulations and even suggest that threats to the self could be a more important cause of reactions to 
experienced fairness than a strong other theoretical account: mortality salience.

The self-threat salience manipulation was constructed in such a way that it paralleled the mortality salience manipulation most commonly used in terror management studies (cf. Arndt et al., 1999; Greenberg et al., 1990; Harmon-Jones et al., 1997; Van den Bos and Miedema, 2000). This made it possible to scientifically investigate the impact of these two salience manipulations.

The self-threat manipulations used in the current research presumably are more precise and more specifically self-related manipulations than mortality salience manipulations, which can evoke thoughts and feelings not only about the participants' own mortality, but also thoughts and feelings concerning other topics (such as uncertainty about the self, or concern about how friends and family will cope, among others). Because mortality salience manipulations are likely to instigate more than self-related feelings and thoughts (see, for instance, McGregor et al., 2001; Mikulincer et al., 2002, Van den Bos et al., 2005), the effects of mortality salience on subsequent fairness manipulations will be not as straightforward and impactful as manipulations that are more directly focused on the self, such as the direct self-threats used in the self-threat conditions of our two experiments.

\section{GENERAL DISCUSSION}

Our line of reasoning was corroborated by the results of two experiments. The findings of Experiment 1 reveal that people react more strongly to fairness manipulations under conditions of self-threat, compared to a nothreat situation. Experiment 2 showed that the effects of self-threat on people's reactions to variations in distributive fairness can be stronger than effects of mortality salience. These are important findings, we think, because for the first time it was shown that a self-threat other than mortality or uncertainty salience, has a strong influence on people's perceptions of fairness.

A close inspection of the findings reported in the current paper shows that in Experiment 1 significant effects of the threat manipulations were found on participants' fairness ratings within the voice, as well as the novoice conditions. In Experiment 2, effects of the salience manipulations did not reach conventional levels of significance within either the equal or unequal outcome conditions. The dissimilarity between these two findings may have been caused by the difference in psychological impact of the different fairness manipulations of Experiments 1 and 2.

People have long been fascinated and have puzzled about the challenging question of why fairness matters. Both experiments reported here 
indicate that self-threats can lead to interaction effects with fairness manipulations, thus providing more insight into why people sometimes react more strongly to variations in perceived fairness.

The findings of the experiments presented here show that self-threats affect reactions to perceived fairness: Asking people to think about being perceived as not intelligent (Experiment 1) or being judged in a negative way (Experiment 2) leads to stronger effects of perceived fairness on fairness judgments and negative affect. Taken together, these experiments reveal that fairness especially matters to people when their selves have been threatened.

In the experiments reported here, we aimed to further specify the general concept of self-related uncertainty as was put forward by Van den Bos (2001) and Van den Bos and Lind (2002). The uncertainty manipulations used in the experiments reported by Van den Bos (2001), for instance, made general personal uncertainties salient. However, as Van den Bos and Lind (2002) pointed out, not all uncertainties are the same. Presumably, making such a broad concept salient may instigate salience of several types of uncertainty, which could range from positive uncertainty (e.g., the thrill of a fair gamble), relatively neutral uncertainty (e.g., pondering on whether to order chicken or beef for lunch), to negative uncertainty (for instance, not knowing whether an authority is to be trusted). These different types of uncertainty cannot be expected to have the same effects, and making the general concept of uncertainty salient could therefore have less distinct effects than the more precise and specifically self-related manipulations used in the current research.

As noted previously in the discussion of Experiment 2, making mortality salient could make other topics salient as well. The effects of mortality salience manipulations on reactions to procedural or distributive fairness may therefore be less clear-cut than the self-threat manipulations used in the current research. This line of reasoning does, of course, not mean that death and mortality are not important, as our findings clearly show strong effects on people's reactions in the mortality salient conditions. We would like to make a plea for broadening the scope to include other threatening issues as well (see also Jost et al., 2003).

Furthermore, we would like to point out that in earlier research on fairness and mortality salience (e.g., Van den Bos and Miedema, 2000; Van den Bos et al., 2005a) and uncertainty salience (Van den Bos, 2001), the main dependent variable were participants' affective reactions toward the way they were treated or the outcome distribution they received. In the experiments described by Van den Bos (2001) and Van den Bos and Miedema (2000) there were no reliable interaction effects on fairness judgments. In the two experiments described above, the main dependent variable was a scale of perceived fairness (Experiments 1 and 2), as well as affect (Experiment 2). The fact that effects on perceived fairness were stronger under conditions of 
self-threat than under conditions of mortality salience is, in our view, supportive of the idea that these effects can be explained as a self-enhancing social psychological process.

The current line of reasoning needs to be corroborated by further studies, in which for instance different dependent variables, and other cultural concepts such as norms and values are used to investigate the relationship between terror management, uncertainty management and the self, (for suggestions, see, e.g., Greenberg et al., 1997; McGregor et al., 2001; Pyszczynski et al., 1999; Solomon et al., 1991; Van den Bos and Lind, 2002), but findings of the studies presented here indicate that concerns for the self are a key antecedent of reactions toward fair and unfair treatment. Terror management theory focuses strongly on the importance of death to account for social psychological effects and argues, among other things, that mortality salience is a very important antecedent of people's reactions toward transgressions and upholding of cultural norms and values (e.g., Greenberg et al., 1997; Pyszczynski et al., 1999; Solomon et al., 1991). On the basis of terror management theory one would therefore be inclined to expect mortality salience to be a prime, perhaps even the main cause of people's reactions to variations in procedural fairness. Reflecting on the findings reported in the current paper the conclusion seems warranted that mortality salience is important in predicting fairness effects, but that a self-threat seems to be even more important.

However, these findings can only be interpreted to predict behavior when someone's self is threatened, and when fairness is used to reduce the threat to this person - and no other. Previous research has proven that people after a mortality salience manipulation show more derogatory evaluations of someone who threatens their beliefs (Greenberg et al., 1990), become more punitive toward moral transgressors (Rosenblatt et al., 1989), see a victim of a crime as more deserving (Landau et al., 2004), or even become more lenient toward a hate-crime perpetrator (Lieberman et al., 2001). In other words, participants in these studies became less fair after a self-threat. How do these results compare to the findings of Experiments 1 and 2? A very important difference between the current studies and the work cited above is that in the previous research, people were reacting toward someone else to reduce the self-threat. Participants in these studies reacted objectively in less fair ways, but reactions to self-threats are probably affective, rather than rational or objective (see also Tesser, 2000).

Miedema et al. (2005) investigated subjective reactions towards fairnessrelated situations that are, objectively, less fair. According to equity theory (Adams, 1965), fairness judgments depend on the comparison of the ratio of one's own inputs and outcomes in a given situation with the inputs and outcomes of another person (or persons) in the same situation, or of oneself in a different situation (see e.g., Adams, 1965; Van den Bos et al., 1997). 
Earlier research on equity theory has shown that equitable outcomes usually evoke positive reactions, and that disadvantageous unequal outcomes mostly result in negative reactions (see, e.g., Austin et al., 1980; Austin and Walster, 1974; Buunk and Van Yperen, 1989; Van den Bos, 1999; Van den Bos et al., 1998). Interestingly, advantageous unequal outcomes are oftentimes also followed by negative reactions, although less strong as compared to disadvantageous arrangements. Because people prefer equity to inequity, it seems as though self-interest is mostly not as important as fairness is.

Miedema et al. (2005) investigated the role of the self in the most interesting equity-related situation: receiving a better outcome than a comparable other person (see also Adams, 1965; Van den Bos et al., 1997). When taking an economic point of view, getting more for the same amount of work seems very nice. However, people usually state that they think being advantaged by inequity is not fair, and they show less positive reactions to advantageous inequity than to equitable outcomes. Findings of both experiments reported by Miedema et al. (2005) indicate that the dislikes of being advantaged in an inequitable way disappear to a great extent when people feel threatened. These results were interpreted to suggest that during the formation of a response to advantageous inequity, fairness considerations become inferior to the tendency of self-enhancement when people feel threatened. As a result, people's reactions, and especially their affect ratings, indicate that in these conditions people pay less attention to fairness. This provides support for the notion that fairness can be used as a means of selfenhancement. People want to act and, also very important, want to be seen as fair and moral (cf. Rivera and Tedeschi, 1976), but when a threatening situation emerges, people appear to care more about themselves than about fairness (see also Van den Bos et al., in press).

The idea that fairness can be used as a self-enhancing mechanism, may be able to explain the seemingly contradicting effects as reported by Brockner et al. (1998), who found that persons with high self-esteem are more influenced by procedural fairness, and Vermunt et al. (2001), who reported that people with low self-esteem are more influenced by procedural fairness. These two studies may refer to different self-related processes. Brockner et al. (1998) manipulated the participants' beliefs about their capability to provide meaningful input. This manipulation of a belief of high capability may have worked as a self-affirmation, which resulted in stronger reactions toward perceived fairness. Studies by Miedema et al. (2005) have shown that affirming the self can indeed result in stronger reactions toward perceived fairness. A belief of being capable of a high level of input could have been self-affirming, and resulting in higher self-esteem, which led to higher satisfaction ratings. Participants who, after the self-affirming high capability manipulation, did not receive an opportunity to voice their opinion reacted by reporting lower levels of self-esteem. 
Participants in the study reported by Vermunt et al. (2001) were prisoners in a correctional facility. Imprisoned people with low self-esteem may be more influenced by procedural justice information because, knowing that in Dutch prisons food, drink, and a place to sleep are guaranteed, they may attempt to enhance (repair) their selves by focusing on procedural issues (which provide clues on status, respect, and standing; see, e.g., Lind and Tyler, 1988; Thibaut and Walker, 1975; Tyler and Lind, 1992). Detainees with high self-esteem do not need to enhance their selves with procedural fairness issues, and thus have a chance to further enhance themselves by material gain (and are thus more influenced by distributive fairness information). Although this line of reasoning is speculative, it seems that the apparently contradicting results as reported by Brockner et al. (1998) and Vermunt et al. (2001) can be explained by looking at the function of the self in fairness perception and the formation of fairness judgments: Brockner et al. (1998) and Vermunt et al. (2001) were tapping into different self-related processes (see also Van den Bos et al., 2005).

We follow Van den Bos and Lind (2002) and Van den Bos et al. (2005a) in suggesting that the management of uncertainty is a very important factor in understanding why fairness matters. However, by showing effects of selfthreat on people's reactions to perceived fairness in ways that we predicted on the basis of important social psychological theories (see, e.g., Baumeister, 1998; Leary and Baumeister, 2000; Koole, 2000; Kuhl, 2000), the present findings provide more specific new insights into the antecedents of reactions to social justice: fairness is more important to people when they feel threatened by self-related uncertainty. The present findings suggest that fairness matters to people when their selves have been threatened. This suggests that having identified the influence of self-threat on reactions to perceived fairness may help scientists in their progress toward understanding why fairness matters.

\section{ACKNOWLEDGMENTS}

This research was supported by the Netherlands Organization for Scientific Research (NWO), project number 400-10-030.

\section{REFERENCES}

Adams, J. S. (1965). Inequity in social exchange. In Berkowitz, L. (ed.), Advances in Experimental Social Psychology, Vol. 2, Academic Press, New York, pp. 267-299.

Arndt, J., Greenberg, J., Solomon, S., Pyszczynski, T., and Schimel, J. (1999). Creativity and terror management: evidence that creative activity increases guilt and social projection following mortality salience. J. Personality Social Psychol. 77, 19-32. 
Austin, W., McGinn, N. C., and Susmilch, C. (1980). Internal standards revisited: effects of social comparisons and expectancies on judgments of fairness and satisfaction. J. Exp. Social Psychol. 16, 426-441.

Austin, W., and Walster, E. (1974). Reactions to confirmations and disconfirmations of expectancies of equity and inequity. J. Personality Social Psychol. 30, 208-216.

Baumeister R. F. (1998). The self. In Gilbert, D., Fiske, S. T., and Lindzey, G. (eds.), Handbook of Social Psychology, Vol. 1, 4th ed. McGraw-Hill, New York, pp. 680-740.

Brockner, J., Heuer, L., Siegel, P. A., Wiesenfeld, B., Martin, C., Grover, S., Reed, T., and Bjorgvinsson, S. (1998). The moderating effect of self-esteem in reaction to voice: converging evidence from five studies. J Personality Social Psychol. 75, 394-407.

Brockner, J., and Wiesenfeld, B. M. (1996). An integrative framework for explaining reactions to decisions: interactive effects of outcomes and procedures. Psychol. Bull. 120, 189-208.

Buunk, B. P., and Van Yperen, N. W. (1989). Social comparison, equality, and relationship satisfaction: gender differences over a ten-year period. Social Justice Res. 3, 157-180.

Cohen, R. L. (1986). Justice: Views from the Social Sciences, New York: Plenum.

De Cremer, van Knippenberg, D., van Dijke, M., and Bos, A. E. R. (2004). How self-relevant is fair treatment? Social self-esteem moderates interactional justice effects. Social Justice Res. 17(4), 407-419.

Folger, R. (1977). Distributive and procedural justice: combined impact of "voice" and improvement of experienced inequity. J. Personality Social Psychol. 35, 108-119.

Folger, R. (1984). Preface. In Folger, R. (ed.), The Sense of Injustice: Social Psychological Perspectives, Plenum Press, New York, pp. ix-x.

Folger, R., and Cropanzano, R. (1998). Organizational Justice and Human Resource Management, Thousand Oaks, CA: Sage.

Folger, R., Rosenfield, D., Grove, J., and Corkran, L. (1979). Effects of "voice" and peer opinions on responses to inequity. J. Personality Social Psychol. 37, 2253-2261.

Greenberg, J., Pyszczynski, T., Solomon, S., Rosenblatt, A., Veeder, M., Kirkland, S., and Lyon, D. (1990). Evidence for terror management theory II: the effects of mortality salience on reactions to those who threaten or bolster the cultural worldview. $J$ Personality Social Psychol. 58, 308-318.

Greenberg, J., Solomon, S., and Pyszczynski, T. (1997). Terror management theory of selfesteem and cultural worldviews: empirical assessments and conceptual refinements. In Zanna, M. (ed.), Advances in Experimental Social Psychology, Vol. 29, Academic Press, New York, pp. 61-139.

Harmon-Jones, E., Simon, L., Greenberg, J., Pyszczynski, T., Solomon, S., and McGregor, H. (1997). Terror management theory and self-esteem: evidence that increased self-esteem reduces mortality threats. $J$ Personality Social Psychol. 72, 24-36.

Jost, J. T., Glaser, J., Kruglanski, A. W., and Sulloway, F. J. (2003). Political conservatism as motivated social cognition. Psychol. Bull. 129, 339-375.

Koole, S. L. (2000). Positivity in Self-Evaluation. Unpublished doctoral dissertation; Nijmegen University, the Netherlands.

Koper, G., Knippenberg, D., Bouhuijs, F., Vermunt, R., and Wilke, H. (1993). Procedural fairness and self-esteem. Eur. J. Social Psychol. 23, 313-325.

Kuhl, J. (2000). A functional-design approach to motivation and self-regulation: the dynamics of personality systems interactions. In Boekaerts, M., Pintrich, P. R., and Zeidner, M. (eds.), Handbook of Self-Regulation, Academic Press, San Diego, pp. 111-169.

Landau, M. J., Johns, M., and Greenberg, J. (2004). A function of form: terror management and structuring the social world. J. Personality Social Psychol. 87, 190-210.

Leary, M. R., and Baumeister, R. F. (2000). The nature and function of self-esteem: sociometer theory. In Zanna, M. P. (ed.), Advances in Experimental Social Psychology, Vol. 32, Academic Press, New York, pp. 1-62.

Lieberman, J. D., Arndt, J., and Personius, J. (2001). Vicarious annihilation: the effect of morality salience on perceptions of hate crimes. Law Human Behav. 25, 547-566.

Lind, E. A., and Tyler, T. R. (1988). The Social Psychology of Procedural Justice, New York: Plenum. 
McGregor, I., Zanna, M. P., Holmes, J. G., and Spencer, S. J. (2001). Compensatory conviction in the face of personal uncertainty: going to extremes and being oneself. J. Personality Social Psychol. 80, 472-488.

Miedema, J., Van den Bos, K., and Vermunt, R. (2005). Exploring when people care less about fairness: the influence of ego threat on reactions to advantageous inequity (submitted).

Miedema, J., Van den Bos, K., Vermunt, R., and Zwenk, F. (2005). On the origins of why fairness matters: The self (submitted).

Mikula, G., and Wenzel, M. (2000). Justice and social conflict. Int. J. Psychol. 35, 126-135.

Mikulincer, M., Florian, V., Birnbaum, G., and Malishkevich, S. (2002). The death-anxiety buffering function of close relationships: exploring the effects of separation reminders on death-thought accessibility. Personality Social Psychol. Bull. 28, 287-299.

Pyszczynski, T. A., Greenberg, J., and Solomon, S. (1999). A dual-process model of defense against conscious and unconscious death-related thoughts: an extension of terror management theory. Psychol. Rev. 106, 835-845.

Rivera, A. N., and Tedeschi, J. T. (1976). Public versus private reactions to positive inequity. J. Personality Social Psychol. 34, 895-900.

Rosenblatt, A., Greenberg, J., Solomon, S., Pyszczynski, T., and Lyon, D. (1989). Evidence for terror management theory I: the effects of mortality salience on reactions to those who violate or uphold cultural values. J Personality Social Psychol. 57, 681-690.

Smith, H. J., Tyler, T. R., Huo, Y. J., Ortiz, D. J., and Lind, E. A. (1998). The self-relevant implications of the group-value model: group-membership, self-worth and treatment quality. J. Exp. Psychol. 34, 470-493.

Solomon, S., Greenberg, J., and Pyszczynski, T. (1991). A terror management theory of social behavior: the psychological functions of self-esteem and cultural worldviews. In Berkowitz, L. (ed.), Advances in Experimental Social Psychology, Vol. 24, Academic Press, New York, pp. $93-159$.

Stroessner, S. J., and Heuer, L. B. (1996). Cognitive bias in procedural justice: formation and implications of illusory correlations in perceived intergroup fairness. J. Personality Social Psychol. 71, 717-728.

Tesser, A. (2000). On the confluence of self-esteem maintenance mechanisms. Personality Social Psychol. Rev. 4, 290-299.

Tesser, A., and Martin, L. L. (1996). The psychology of evaluation. In Higgins, E. T., and Kruglanski, A. W. (eds.), Social Psychology: Handbook of Basic Principles, Guilford, London, pp. 400-432.

Thibaut, J., and Walker, L. (1975). Procedural Justice: A Psychological Analysis, Hillsdale, NJ: Erlbaum.

Tyler, T. R. (1990). Why Do People Obey the Law? Procedural Justice, Legitimacy, and Compliance, New Haven, CT: Yale University Press.

Tyler, T. R. (1997). The psychology of legitimacy: a relational perspective on voluntary deference to authorities. Personality Social Psychol. Rev. 1, 323-345.

Tyler, T. R., and Smith, H. J. (1998). Social justice and social movements. In Gilbert, D., Fiske, S. T., and Lindzey, G. (eds.), Handbook of Social Psychology, Vol. 4, McGraw-Hill, Boston, MA, pp. 595-629.

Tyler, T. R., and Lind, E. A. (1992). A relational model of authority in groups. In Zanna, M. P. (ed.), Advances in Experimental Social Psychology, Vol. 25, Academic Press, San Diego, CA, pp. 115-191.

Van den Bos, K. (1999). What are we talking about when we talk about no-voice procedures? On the psychology of the fair outcome effect. J. Exp. Social Psychol. 35, 560-577.

Van den Bos, K. (2001). Uncertainty management: the influence of uncertainty salience on reactions to perceived procedural fairness. J Personality Social Psychol. 80, 931-941.

Van den Bos, K., and Lind, E. A. (2002). Uncertainty management by means of fairness judgments. In Zanna, M. P. (ed.), Advances in Experimental Social Psychology, Vol. 34, Academic Press, San Diego, CA.

Van den Bos, K., Lind, E. A., Vermunt, R., and Wilke, H. A. M. (1997). How do I judge my outcome when I do not know the outcome of others? The psychology of the fair process effect. J. Personality Social Psychol. 72, 1034-1046. 
Van den Bos, K., and Miedema, J. (2000). Toward understanding why fairness matters: the influence of mortality salience on reactions to procedural fairness. J. Personality Social Psychol. 79, 355-366.

Van den Bos, K., Peters, S. L., Bobocel, D. R., and Ybema, J. F. (in press). On ethical considerations and egoism-based pleasure: reactions to advantageous inequity under cognitively busy conditions. J. Exp. Social Psychol.

Van den Bos, K., Poortvliet, P. M., Maas, M., Miedema, J., and Ham, E.-J. (2005a). An enquiry concerning the principles of cultural norms and values: the impact of uncertainty and mortality salience on reactions to violations and bolstering of cultural worldviews. J. Exp. Social Psychol. 41, 91-113.

Van den Bos, K., Steiner, D. D., van Yperen, N. W., and Dekker, D. M. (2005b). Culture and the Psychology of Voice: The Moderating Influence of Performance Goal Orientation (submitted).

Van den Bos, K., Vermunt, R., and Wilke, H. A. M. (1996). The consistency rule and the voice effect: the influence of expectations on procedural fairness judgments and performance. Eur. J. Social Psychol. 26, 411-428.

Van den Bos, K., Wilke, H. A. M., Lind, E. A., and Vermunt, R. (1998). Evaluating outcomes by means of the fair process effect: evidence for different processes in fairness and satisfaction judgments. J. Personality Social Psychol. 74, 1493-1503.

Vermunt, R., Knippenberg, D., Knippenberg, B., and Blaauw, E. (2001). Self-esteem and outcome fairness: differential importance of procedural and outcome considerations. J. Appl. Psychol. 68, 621-628.

Watson, D., Clark, L. A., and Tellegen, A. (1988). Development and validation of brief measures of positive and negative affect: the PANAS scales. J. Personality Social Psychol. 54, 1063-1070. 